\title{
A Multi-Objective Extremal Optimisation Approach Applied to RFID Antenna Design
}

\author{
Pedro Gómez-Meneses, Marcus Randall and Andrew Lewis
}

\begin{abstract}
Extremal Optimisation (EO) is a recent nature-inspired meta-heuristic whose search method is especially suitable to solve combinatorial optimisation problems. This paper presents the implementation of a multi-objective version of EO to solve the real-world Radio Frequency IDentification (RFID) antenna design problem, which must maximise efficiency and minimise resonant frequency. The approach we take produces novel modified meander line antenna designs. Another important contribution of this work is the incorporation of an inseparable fitness evaluation technique to perform the fitness evaluation of the components of solutions. This is due to the use of the NEC evaluation suite, which works as a black box process. When the results are compared with those generated by previous implementations based on Ant Colony Optimisation (ACO) and Differential Evolution (DE), it is evident that our approach is able to obtain competitive results, especially in the generation of antennas with high efficiency. These results indicate that our approach is able to perform well on this problem; however, these results can still be improved, as demonstrated through a manual local search process.
\end{abstract}

Pedro Gómez-Meneses

School of Information Technology, Bond University, QLD 4229, Australia, e-mail: pedgomez e bond. edu. au

Departamento de Ingeniería Informática, Universidad Católica de la Santísima Concepción, Concepción, Chile, e-mail: pgomez@ucsc.cl

Marcus Randall

School of Information Technology, Bond University, QLD 4229, Australia, e-mail: mrandalle bond.edu. au

Andrew Lewis

Institute for Integrated and Intelligent Systems, Griffith University, Queensland, Australia, e-mail: a. lewis@griffith.edu. au 


\section{Introduction}

RFID [7] is the technology used to automatically identify objects at a distance without any physical or sight contact. RFID is widely used in applications such as smart cards, electronic passports, access control, container identification, animal identification, sporting events, medical applications and industrial automation [5].

The RFID implementation requires a tag, which consists of a microchip together with a radio antenna. For a description of the principles of RFID, the reader is referred to $[6,7,8,12,14]$. The design of the antenna, as is demonstrated in the new section, is a Multi-Objective Combinatorial Optimisation Problem (MOCOP) [3]. Until recently, engineers primarily designed these by hand; however, nature-inspired meta-heuristics have started to be used to try to find approximated optimal configurations in the design of RFID antennas. There is an initial and isolated work with genetic algorithms [10], which was centred on a simple and regular RFID antenna design. A more systematic and general investigation has been developed on the design of meander line antennas using Ant Colony Optimisation (ACO) [8, 9, 12, 13, 14] and Differential Evolution (DE) [11]. The research with ACO and DE has demonstrated that it is possible to apply nature-inspired search mechanisms to design and evaluate meander line RFID antennas.

The above has motivated the development of an application for the design of RFID antennas based on EO [1]. EO works by selecting one of the components that make up the sole solution used in the algorithm. This selection is performed by the assessment of the contribution of each component of the solution. Hence, the component that degrades the solution evaluation the most has the highest probability of being chosen. Its value will be then replaced by a different value. Over a number of iterations, EO will gradually improve the quality of solutions it generates. The implementation of the EO approach for this problem is an interesting challenge because the evaluation for any valid solution of an antenna layout is performed by an external black-box module provided by the free antenna modelling software package, NEC [2]. In terms of the meta-heuristic, there is no information about the potential value of components with which to guide the search. Therefore, an inseparable fitness evaluation mechanism is required to be implemented. One such scheme is developed as part of this research.

The inseparable fitness evaluation technique will be based on the idea of the global pheromone scheme used in ACO. This scheme will serve to maintain a record, over generations, of the components present in the solutions that have a good evaluation. Thus, components with a higher presence in good solutions will have a higher level of pheromone, which means that these components will be more likely not to change as a result of EO's selection mechanism.

The creation of a meander line is the most common form to design a RFID antenna. The antenna is a folded dipole that can be represented by a Cartesian grid $[6,12]$ (see Figure 1), which is symmetrical around the dipole. The grid is comprised of a finite number of points in a square shape. The meander line is created by the connection of these points by horizontal or vertical segments, so that no segment intersects another already established. It is not mandatory that all grid points 


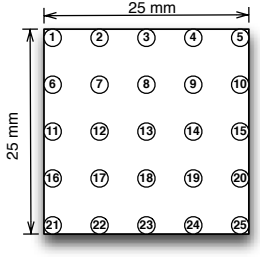

(a)

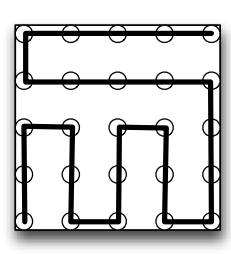

(b)

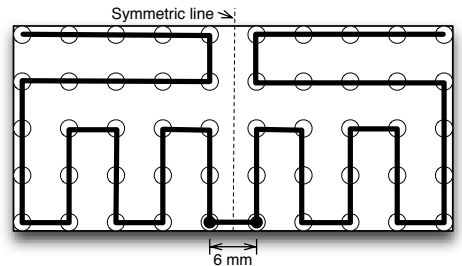

(c)

Fig. 1 (a) The grid defines a $5 \times 5$ antenna. (b) Illustrates a potential meander line antenna for the grid defined in (a). (c) The dipole antenna generated through the binding of two mirrored meander line antennas shown in (b) by a $6 \mathrm{~mm}$ join.

must be connected; however, the meander line must start on any adjacent point to the symmetric line that divides the two halves of the antenna. The start point is used to connect, with an additional line, the two-mirrored dipoles of the antenna.

Recently, Randall et al. [12] developed an initial software system to design and evaluate the efficiency of meander line RFID antennas based on ACO. The approach was successfully applied on grids from size $5 \times 5$ grid points to $10 \times 10$ as a constructive mechanism creating a path on the grid. Continuing this research, Weis et al. [13] proposed an extension based on a local search technique that uses an operator known as backbite. The next step was developed by Lewis et al. [8, 9] who proposed a multi-objective version, which maximises the efficiency and minimises the resonant frequency. After that, Weis et al. [14] proposed a mechanism to increment the performance of the ACO software using an initial pheromone biased by the a-priori knowledge from human designers. Each one of this works show an improvement for all grid instances compared to those obtained by the previous work.

Montgomery et al. [11] developed a multi-objective DE approach, which differs from ACO in the way the solution is created. Instead of using a constructive mechanism, the DE algorithm starts with a complete initial solution, which is iteratively improved using vector differences to modify it. The results showed that DE is an appropriate method to achieve good solutions, being more effective for smaller antenna grid sizes. The DE approach did not use any local search mechanism to improve the solutions. The DE approach demonstrates that it is possible to implement a non-constructive technique to solve the RFID antenna design problem, which is inherently constructive in nature. However, DE is a population-based search method suitable to solve continuous optimisation problems and the RFID antenna design problem has been modelled as a combinatorial problem. For this reason, it is believed that the non-constructive EO meta-heuristics, whose search methods is especially suitable for solving combinatorial optimisation problems, could also achieve competitive results to those generated by the ACO approach.

The EO approach described in this paper is tested and compared on the same grid sizes as those used Lewis et al. [9]'s multi-objective ACO approach and Montgomery et al. [11]'s multi-objective DE approach.

The rest of this paper is organised as follows. Section 2 explains the inseparable fitness evaluation scheme to solve problems that do not provide the necessary 
information to perform a separable evaluation of components. Section 3 presents the extremal optimisation approach applied to the RFID antenna design problem. Section 4 gives a summary of the computational experiments developed with an analysis of them. Section 5 presents the conclusions and discusses the future work arising from this study.

\section{Inseparable Fitness Evaluation Scheme}

In EO, each component of the solution is evaluated to assess its contribution to the quality of the optimal. This evaluation is done at every iteration and it is essential that the problem to be solved has as much suitable information as possible for this purpose. However, not all problems can provide this information. For this reason, it is necessary to incorporate a mechanism into EO in order to solve problems where it is not possible to carry out separable component fitness evaluations.

In the RFID antenna design problem, the input data are: the grid size, the side antenna size, the wire width and the dipole segment connection length. This information, along with a proposed antenna design, is used as input to the NEC software. This software works as a black box computation, which returns the evaluation with regard to the efficiency and the resonant frequency. No other data is available or known about these calculations, which may be used to perform the component fitness evaluation.

The inseparable fitness evaluation scheme proposed herein is based on the pheromone structures used by Ant Colony System (ACS) [4]. In ACS, each time an ant traverses a path from the nest to the food source, a local pheromone update is done. This action is performed until a complete route is produced, updating the pheromone level at each section of the route. Once all the ants have finished the creation of a route from the nest to the food source, a second update is carried out. Here, the best route found at the current iteration is used as a global pheromone update for each section in this route. As EO is not a method that generates a solution by applying a constructive mechanism, only the global pheromone update is considered.

The idea behind the use of the pheromone structure is motivated by the fact that it works as an element which can keep a record of the solution components that are present in good solutions. In this way, each time a new non-dominated solution is found, then a pheromone update is performed. With this procedure, the components that degrade the solution will have low pheromone levels and the component that contributes to the optimality of the solution will have a high pheromone level. Thus, the pheromone structure allows the implementation of a fitness evaluation of the components of the solution without any specific information about the evaluation of the objective function(s).

In the particular case of the RFID antenna design problem, each component of the pheromone structure represents a segment that joins two adjacent non-diagonal points of the grid. The collection of these segments composes one half of the sym- 


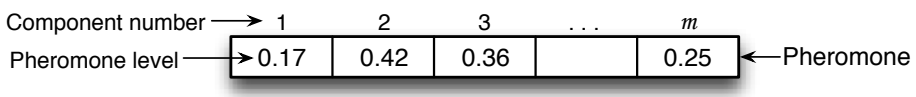

Fig. 2 An example of a pheromone vector for the RFID antenna design problem, where $m$ is the number of components in the solution. $m$ is formally defined in the next section.

metrical design. Figure 2 shows a pheromone illustration for the RFID antenna design problem.

The pheromone vector is initialised with a very low value between zero and one. After that, the pheromone level for each component is updated along the extremal optimisation search process according to Equation 1.

$$
\Phi_{i}^{t}=(1-\alpha) \Phi_{i}^{t-1}+\alpha \Delta
$$

where:

$\Phi_{i}^{t}$ is the pheromone value for the $i^{\text {th }}$ component at time $t$,

$\alpha$ is the pheromone decay factor, and

$\Delta$ is the pheromone positive reinforcement for the $i^{\text {th }}$ component if it belongs to a non-dominated solution, 0 otherwise.

This pheromone update is applied only to each non-dominated solution found by EO and only the components that have allocated a segment that belongs to the solution are able to obtain this reinforcement. Finally, the inseparable fitness evaluation scheme of a solution is given by Equation 2.

$$
\lambda\left(x_{i}\right)=\Phi\left(x_{i}\right), \quad \forall i \quad 1 \leq i \leq n
$$

where:

$\lambda\left(x_{i}\right)$ is the fitness evaluation for the $i^{\text {th }}$ component of the solution,

$\Phi\left(x_{i}\right)$ is the pheromone level for the $i^{\text {th }}$ component of the solution,

$n \quad$ is the number of components of the solution.

Note that the fitness function evaluation, for each component of the solution, is completely defined by the pheromone structure. It is unnecessary to have any other information with respect to this particular problem. This scheme is very general and applicable to other black box problems.

\section{EO Applied to RFID Antenna Design}

Until now, all approaches based on either ACO or DE, have worked with a meander line antenna design. These works fulfilled the objective of demonstrating that it possible to develop a software system to design and evaluate meander line RFID antennas using both a constructive and a non-constructive meta-heuristics. How- 


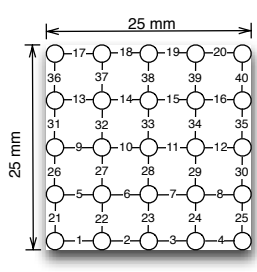

(a)

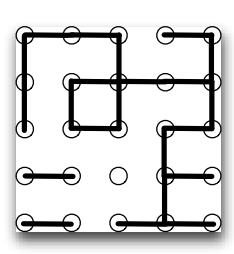

(b)

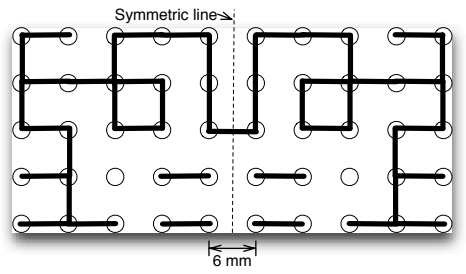

(c)

Fig. 3 (a) The grid representation for a $5 \times 5$ antenna based on segment allocation. (b) Illustrates a potential modified meander line. (c) The dipole antenna generated with two mirrored modified meander lines connected by a $6 \mathrm{~mm}$ segment.

ever, new questions emerge as to the possibility of applying other nature-inspired meta-heuristics for generating antenna designs and if it is possible to build efficient antennas with a different design to that of a meander line.

In response to these questions, the idea of developing an EO approach using modified meander lines, is proposed. Here, the restriction of generating a single continuous line is relaxed and now a line with loops, mesh segments and parasitic elements can be designed. This revised scenario involves a new interpretation of how the grid is built.

In previous works, each node on the grid is tagged with an identification number from 1 to $n^{2}$, where $n$ is the grid size, as was shown in Figure 1. Therefore, the solution is represented by a sequence of nodes, which are joined in order by a continuous line to generate the antenna design. However, in a modified meander line antenna design scheme, the same grid has a different interpretation. Here, the segments that join the nodes are tagged with an identification number from 1 to $m$, where $m=(2 \times(n-1) \times n)$, as shown in Figure 3 .

For the modified meander line case, the solution is represented by a vector of $m$ components. Each component represents a segment that could potentially be put in the antenna design. If the $i^{\text {th }}$ segment is allocated to the antenna design, then a value of 1 is assigned to its position in the solution vector. A value of 0 is assigned to signify that the segment is not part of the antenna design (see Figure 4).

This representation to design a general modified meander line antenna becomes a combinatorial optimisation problem similar to the knapsack problem. The main difference between this and the problem defined in this paper, is that the weight for each knapsack item is known, whereas in the modified meander line antenna design there is no information related to any sort of weight that is associated with the segments. Also, there is the constraint that a segment associated to the edge of



Fig. 4 An example of a solution vector for the modified meander line antenna. 
the grid aligned to the symmetric line, must be allocated. Taking into consideration these differences, the modified meander line antenna design will be formulated as a multi-objective knapsack problem.

The initial solution is obtained by a random selection of segments. This mechanism could generate an infeasible solution due to the requirement that at least one segment associated to the edge of the grid aligned to the symmetric line must be in the antenna design. This is because the additional $6 \mathrm{~mm}$ segment is used to connect and energise the two-mirrored grids that form the dipole antenna. If an initial feasible solution is generated then this is added as the first point in the approximated Pareto-front set.

For this particular problem, which has no available information to perform an assessment of the components of the solution, a differentiated fitness evaluation is implemented with the support of the inseparable fitness evaluation scheme described in Section 2. Thus, Equation 3 illustrates the fitness evaluation to select the segment that degrades the solution to be assigned or unassigned from the antenna design.

$$
\lambda\left(x_{i}\right)= \begin{cases}\Phi\left(x_{i}\right), \forall i \quad 1 \leq i \leq m & \text { for feasible solutions } \\ -V_{i}, \forall i \in \tilde{M} & \text { for infeasible solutions }\end{cases}
$$

where:

$\Phi\left(x_{i}\right)$ is the pheromone level for the $i^{\text {th }}$ component of the solution,

$m$ is the number of components of the solution,

$V_{i} \quad$ is the number of segments connected to the $i^{\text {th }}$ unassigned segment associated to the edge of the grid aligned to the symmetric line, and

$\tilde{M} \quad$ is the set of segments associated to the edge of the grid aligned to the symmetric line.

When the solution is feasible, the inseparable fitness evaluation based on the pheromone structure is applied to define which components of the solution degrade the solution. Thus, segments that have a low level of pheromone are chosen with a higher probability to be allocated/unallocated than those with a high level of pheromone. This is because a segment with a low level means that it does not contribute to achieving a high efficiency with a low resonant frequency in the modified meander line antenna design. On the other hand, when the solution is infeasible, this means that none of the segments on the symmetric line are allocated. To obtain a feasible solution again, one of them must be selected to be part of the antenna design. Hence segments on the symmetric line, with a high number of neighbouring segments connected to it, are considered to be the components that degrade the solution. This is because a segment that is connected to other adjacent segments has a higher probability of generating a longer continuous line. This is important due to longer continuous lines reducing resonant frequency.

The Roulette Wheel Selection (RWS) scheme is used to select one of the worst evaluated segments according to its probability $P$. The replacement of the value for the chosen component (segment) is a simple process because it only has to change its value from 0 to 1 or from 1 to 0 , as appropriate. 
Due to the very time consuming process to evaluate the solution by the NEC software, for now, secondary search is not performed. However, a local search performed manually, to discern a possible future implementation of this, is carried out. For the same reason, as in previous works $[9,8,11,12,13,14]$, a caching system is implemented to reduce the number of evaluations made by the NEC software and so to reduce the runtime of the EO process.

Each time a feasible solution is found, the non-dominated procedure is activated to update the approximated Pareto-front set found at the current iteration. If a new non-dominated solution is found, then the pheromone level update is performed. The EO procedure is repeated for a pre-set number of iterations. Finally, the approximated Pareto-optimal and Pareto-front sets that were found by the approach are returned as output. Algorithm 1 shows the EO pseudocode for the bi-objective modified meander line antenna design problem.






\section{Computational Experiments}

The EO algorithm was coded in the $\mathrm{C}$ language and compiled with gcc. The tests were performed on a Linux workstation with a $3 \mathrm{GHz}$ Core2 Duo CPU and $3.8 \mathrm{~GB}$ of RAM. The NEC software was the version available in late October 2010.

Test data were the same as used in the previous works by Lewis et al. [8] and Montgomery et al. [11]. These tests consist of six grids, from size $5 \times 5$ to $10 \times 10$. All grids have a dimension of $25 \times 25 \mathrm{~mm}$ and the segment width is $1 \mathrm{~mm}$. An extra segment of $6 \mathrm{~mm}$ in length is used to connect the dipole.

The number of iterations for the search was set to 1000. A higher number of iterations were not performed because the NEC software takes a considerable amount of runtime. Each instance was run three times using a different random seed. Grids from $5 \times 5$ to $7 \times 7$ ran an extra search with 2000 and 3000 iterations only to observe if lower resonant frequencies could be reached.

The initial pheromone level and the pheromone decay factor were set at 0.1 , as these values have been found to be robust by Dorigo and Gambardella [4]. The pheromone positive reinforcement was set at 1.0 as a constant value. This value is calculated as $1 / L$, where $L$ is the length of the best path generated by the ants. But, as EO does not generate a path, this value was implemented as a constant value.

\subsection{Comparison of Results}

The EO results were compared with those obtained by the ACO $[9,8]$ and the DE [11] algorithms. These variants are denoted as $A C O_{q 1}, A C O_{q 5}, A C O_{q 9}, D E$, $D E_{\min L}$. The $q$ index for the ACO approaches refers to three different levels of greediness to determine the next component to add in its constructive process; that is, the probability that a greedy decision is made instead of a probabilistic one. On the other hand, the DE variant $\min L$ alludes to the incorporation of a minimum length constraint in the meander line to encourage the exploration of solutions with lower resonant frequencies.

The objective of this comparative study is to discuss the strengths and weaknesses of the EO approach and the contribution that this new technique for RFID antenna design can provide. To do this, it is necessary to consider the following implementation differences that affect this comparison:

- The ACO and DE approaches work under the restriction of generating meander lines, which can have different lengths. The EO algorithm, however, develops an antenna based on the presence or absence of the segments that make up the grid through a modified meander line that is able to create any design.

- The connection line is another aspect that has been dealt with in different ways. The DE approach always makes the connection line, which is the the link connecting the two halves of the dipole antenna, at node 1, while the ACO and EO algorithm may make the connection in any node on the symmetrical line. 
- The local search mechanism has only been implemented by the ACO approach through the application of the backbite operator [13]. The DE and EO algorithms have not yet implemented a local search mechanism.

- The number of solutions produced by DE and ACO were 10100 and 10000 solutions respectively. However, EO produced only between 1000 and 3000 solutions according to the performed experiment, due to the limited time and available computational resources.

- DE and ACO, are population-based techniques. The variability of new solutions generated by population-based methods is generally greater than the singleindividual-based methods, as is the case for extremal optimisation.

Figure 5 shows a the plotted graph results for the ACO, DE and EO approaches for the grid size $7 \times 7$. The remaining plotted graphs can be obtained from http: //dl.dropbox.com/u/69201895/Evolve2012/index.html.

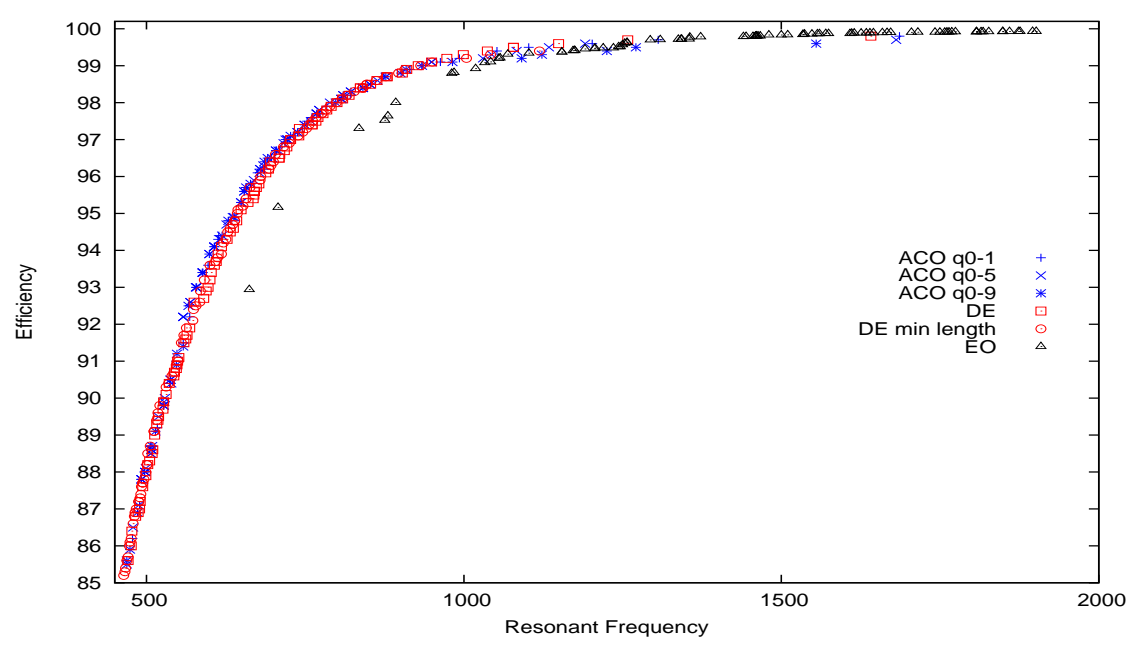

Fig. 5 Result for the $7 \times 7$ bi-objective RFID antenna design problems. This graph is indicative of the performance of $\mathrm{EO}$, regardless of grid size.

The first issue that can be appreciated in the graph is an approximated Paretofront set that is biased towards the objective related to efficiency. This can be interpreted as EO generates designs with a high efficiency instead of antenna designs with low resonant frequencies. This is evident by observing the number of solutions generated in the frequency range $1 \mathrm{GHz}$ to $2 \mathrm{GHz}$. Furthermore, most of the solutions generated in that frequency range achieved better efficiency than those generated by the previous approaches.

Use of the S-metric [15] and the C-metric [15] can achieve a more analytical comparison than the visual analysis of the plotted graphs. The S-metric evaluates how much of the multi-objective space is dominated by a given approximated 


\begin{tabular}{|c|c|c|c|c|c|c|}
\hline Grid Size & $A C O_{q 1}$ & $A C O_{q 1}$ & $A C O_{q 9}$ & $D E$ & $D E_{\min L}$ & $E O$ \\
\hline \hline $5 \times 5$ & 49331.8 & 48658.8 & 48600.0 & 49326.1 & 48053.9 & 49422.6 \\
\hline $6 \times 6$ & 86862.4 & 86557.3 & 86081.1 & 86949.5 & 86898.8 & 86319.2 \\
\hline $7 \times 7$ & 48587.1 & 48333.5 & 48005.2 & 48583.5 & 47525.4 & 47118.1 \\
\hline $8 \times 8$ & 27738.0 & 27738.0 & 27473.7 & 27684.6 & 27201.7 & 19713.8 \\
\hline $9 \times 9$ & 48250.1 & 48177.5 & 47847.0 & 47835.6 & 46291.9 & 36255.8 \\
\hline $10 \times 10$ & 47477.0 & 47333.5 & 47303.5 & 46678.4 & 44753.3 & 35810.6 \\
\hline
\end{tabular}

Table 1 The S-metric values for the RFID antenna design with a grid size of $5 \times 5,6 \times 6,7 \times 7$, $8 \times 8,9 \times 9$ and $10 \times 10$.

Pareto-front set. The C-metric compares two approximated Pareto-front sets through measuring the proportion of points in one set that are dominated by the other set.

From Table 1 it can be observed that EO achieved a similar coverage of the dominated space in comparison with the other approaches for the grids of size $5 \times 5$, $6 \times 6$ and $7 \times 7$. However, for the grids of size $8 \times 8,9 \times 9$ and $10 \times 10$, the S-metric indicated a lower dominated space than the ACO and DE approaches. The decrease in the value of the metric for the three largest grids was due to the reduced number of iterations that EO carried out (just 1000, versus 3000 performed by the three smaller grids). This demonstrates that EO is able to find new, non-dominated solutions as the number of iterations is increased. It is important to note that despite the similarity of the S-metric value achieved by it, the approximated Pareto-front sets obtained by EO is more inclined towards higher resonant frequencies than lower ones.

The C-metric values shown in Table 2 illustrate that even with the low number of iterations performed by EO, the proportion of solutions found for this, that are dominated by the other approaches, is less than $32 \%$. Even further, this became zero in the case of the $9 \times 9$ grid. These values can be considered to be good, but it is

\begin{tabular}{|c|c|c|c|c|c|c|c|c|c|c|c|c|c|}
\hline $5 \times 5$ & & & & 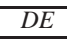 & $D E$ & & $6 \times 6$ & $O_{c}$ & 4 & & $D E$ & $D E$ & \\
\hline$A C O_{q 1}$ & & 0.290 & 0.347 & 0.22 & $\overline{0.1}$ & 0.279 & $\overline{A C O} O_{q 1}$ & & 0.304 & & 0.1 & 0. & .16 \\
\hline $\mathrm{CO}_{q 5}$ & 08 & - & 0.15 & 0.044 & 0. & 0.102 & $\mathrm{CO}_{q 5}$ & .146 & - & 4 & 171 & & .1 \\
\hline$C O_{q 9}$ & 0 & 0.036 & 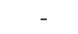 & 0.044 & 0.044 & .102 & $C O_{q 9}$ & 08 & 0.130 & - & 0.105 & 34 & .1 \\
\hline$D E$ & 027 & 0.2 & .173 & - & 0.0 & .205 & $D E$ & 333 & 391 & .519 & & 0.207 & .27 \\
\hline$O E_{\min L}$ & 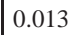 & 0 & 52 & 0.044 & - & 0.205 & $D E_{\min L}$ & 293 & 91 & 80 & 157 & & 0.25 \\
\hline$E O$ & 027 & 0.109 & 0.065 & 0.066 & 0.022 & - & $E O$ & 0.04 & 0.072 & 0.038 & 0.013 & 0.024 & - \\
\hline $7 \times 7$ & $\overline{C O_{q 1}}$ & $A C O_{q 5}$ & $A C O_{q 9}$ & $\overline{D E}$ & $D E_{m}$ & $\overline{E O}$ & $8 \times 8$ & $A C O_{q 1}$ & $A C O_{q 5}$ & $\overline{A C O_{q 9}}$ & $\overline{D E}$ & $D E_{\min L}$ & $\overline{E O}$ \\
\hline$C O_{q 1}$ & & 0.285 & 0.229 & 0.559 & 0.3 & 0.301 & $\overline{A C O_{q 1}}$ & 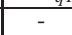 & 0.0 & 0.517 & 0.68 & 0.656 & $\overline{0.066}$ \\
\hline$O_{q 5}$ & 15 & - & 0.208 & 0.623 & 0.479 & 0 & $C O_{q 5}$ & 0.0 & - & 17 & 0. & 56 & .06 \\
\hline$O_{q 9}$ & 210 & 0.116 & - & 0.537 & 0.3 & 87 & $A C O_{q 9}$ & .173 & 0.1 & & 0.65 & 0.585 & .01 \\
\hline & 333 & 181 & .187 & - & 0.173 & 0.311 & $D E$ & 269 & 0.2 & 0.344 & & 0.474 & .08 \\
\hline$E_{\min L}$ & & 0 & 0 & 0.731 & & .16 & $D E_{m}$ & .057 & 0. & 0.241 & 0. & & .01 \\
\hline & 17 & 0.025 & 0.104 & 0.010 & 0.0 & - & $E O$ & 0.038 & 0.038 & 0.137 & 0.011 & 0.0 & 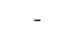 \\
\hline $9 \times 9$ & $A C O_{q 1}$ & $A C O_{q 5}$ & $A C O_{q 9}$ & $\overline{D E}$ & $D E_{\text {min }}$ & $E O$ & $\mathbf{1 0} \times \mathbf{1 0}$ & $A C O_{q 1}$ & $A C O_{q 5}$ & $A C O_{q 9}$ & $\overline{D E}$ & $D E_{\min L}$ & $E O$ \\
\hline$\overline{C O_{q 1}}$ & & 0.573 & 0.608 & 0.824 & 0.9 & 0.051 & $\overline{A C O} O_{q 1}$ & & 0.408 & 0.701 & 0.886 & 1.0 & 0.112 \\
\hline $\mathrm{CO}_{q 5}$ & 166 & - & 0.540 & 0.72 & 0.965 & 0.038 & $C O_{q 5}$ & 0. & - & 0.656 & 0.80 & 1.0 & 0.10 \\
\hline $4 C O_{q 9}$ & 102 & 0.1 & - & 0.662 & 0.8 & 0.0 & $A C O_{q 9}$ & 0.123 & 0.098 & & 0.647 & 0.95 & 0.090 \\
\hline & 166 & 0.253 & 297 & - & 0.87 & 0.102 & & 0.109 & 0.197 & 0.388 & - & 1.0 & 0.127 \\
\hline$E_{\min L}$ & & & 148 & 0.094 & 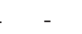 & 0.0 & $D E_{m i}$ & 0.0 & 0.0 & 0.014 & 0.0 & - & 0.05 \\
\hline 50 & 5 & 0.013 & 094 & 0.0 & 0.017 & & $E O$ & 0.01 & 0.014 & 0.029 & 0.01 & 0.033 & 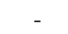 \\
\hline
\end{tabular}

Table 2 The C-metric values for the RFID antenna design with a grid size of $5 \times 5,6 \times 6,7 \times 7$, $8 \times 8,9 \times 9$ and $10 \times 10$. 
necessary to develop a deeper analysis to understand why these values are received. A possible reason for these results is because of the fact that EO creates a type of antenna with different features to those generated by the previous works.

\subsection{Manual Local Search Analysis}

Previous work leant towards the creation of antennas through a continuous line that follows the pattern of a meander path. Generally, the longer the continuous line, the lower the resonant frequency and quicker the NEC evaluation. However, the EO implementation investigates the free allocation of segments in the grid generating antenna designs that do not necessarily follow any predetermined pattern. Therefore, the probability of creating a continuous long line is lower. This feature has a strong impact on both increasing evaluation runtime for the NEC software and decreasing generation of antennas with lower resonant frequencies.

Results showed that EO was able to generate similar approximated Pareto-front sets for runs with different initial seeds. Also, when the iterations are increased, the approach is able to find points on the attainment surface with better convergence and diversity. However, this improvement was not expected to reach solutions with lower resonant frequencies.

To improve the convergence towards lower resonant frequencies, a potential local search mechanism was analysed. The backbite local search mechanism used in previous works cannot be applied to the EO approach as this technique is oriented to the construction of Hamiltonian walks as the case of a meander line. However, EO needs of a mechanism to generate, in the current modified meander line solution, a continuous line as long as possible through the modification of one or more segments. The difficulty of performing this simple operation lies in the modified meander line generated by EO not following any predefined pattern such as a meander line (see Figure 3). The drawn line on the grid can simultaneously produce continuous lines, branched lines, circuits and completely isolated lines.

A heuristic that can be followed is to identify all end segment lines and to try to add a new segment without creating a circuit. For example, Figure 6 (a) shows the minimum resonant frequency $(\mathrm{RF})$ with its respective efficiency $(\mathrm{E})$ that was found in the $5 \times 5$ grid. Here, all end segment lines that potentially can add an adjacent segment are identified. From these points, only segments 31 and 37 are able to add a new segment without generating a circuit. Figure 6 (b) shows the result when segment 32 is incorporated. It can be seen that the resonant frequency decreases, but also there is a decrease in efficiency. A better resonant frequency is achieved when segment 9 is added (see Figure 6 (c)). This improvement is because the incorporation of segment 9 achieves a longer continuous line.

A special case can be given in Figure 7 (a), which illustrates when it is not possible to add an adjacent segment without generating a circuit. Here, there is the option of removing any of the branched segment lines as can be seen in Figure 7 (b). The elimination of one of these branched segment lines achieved a fall in the resonant 

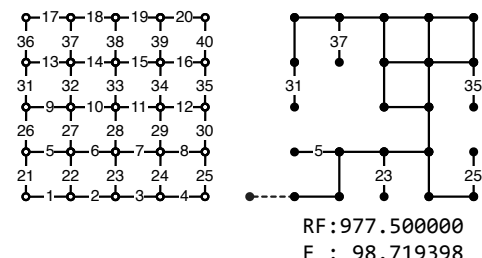

(a)

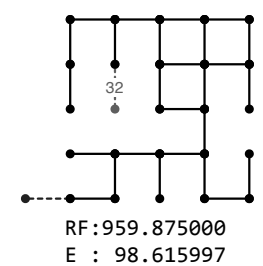

(b)

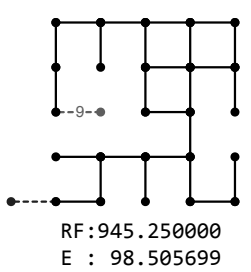

(c)

Fig. 6 A manual local search case for the $5 \times 5$ grid, when it is possible to add a new segment without generating a circuit. The dashed segment represents the connection line.
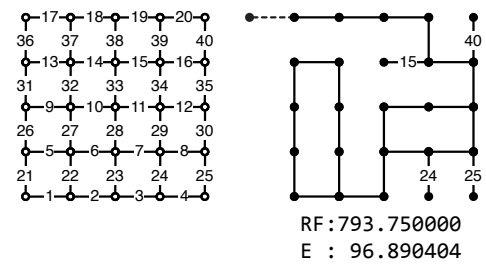

(a)

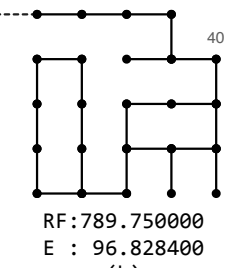

(b)

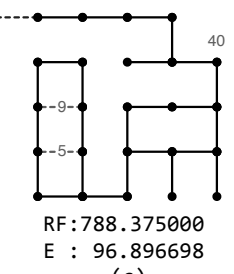

(c)

Fig. 7 A manual local search case for the $5 \times 5$ grid, when it is not possible to add a new segment without generating a circuit. The dashed segment represents the connection line.
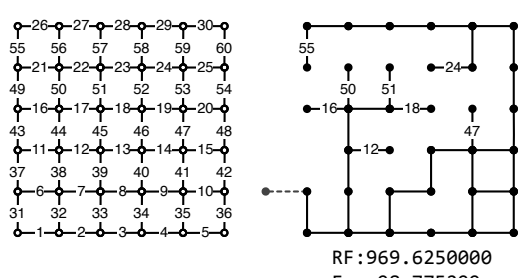

(a)

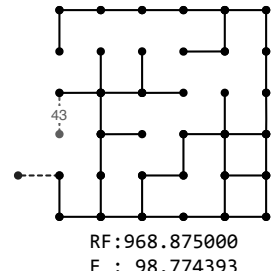

(b)

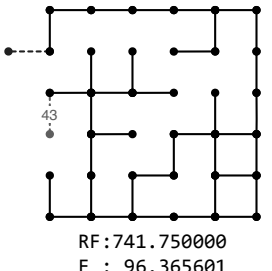

(c)

Fig. 8 A manual local search case for the $6 \times 6$ grid, given a change to the connection line to a different segment in the symmetrical line side. The dashed segment represents the connection line.

frequency. This is an interesting finding that requires more analysis; however, this was not the only discovery. It is possible to reduce the resonant frequency even more with a slight increase in efficiency, which is desirable in RFID. This is possible when segment 40 is eliminated and segments 5 and 9 are incorporated into the circuit that is generated at the end of the continuous segment starting from the line of connection (see Figure 7 (c)). For these two last cases, it is necessary to create a general local search heuristic that allows the discovery of new non-dominated points towards lower resonant frequencies for the antenna design generated by EO.

A third interesting case to discuss is given by analysing the solution with the lowest resonant frequency obtained by EO for the grid size $6 \times 6$. Figure 8 (a) shows the antenna design and identifies the end segment lines that potentially could add an adjacent segment. From these segments, the only one that can add a new segment without generating a circuit is segment 16 . When segment 43 is added to segment 
16, the resonant frequency has a slight decrease from $969.625 \mathrm{MHz}$ to $968.875 \mathrm{MHz}$ (see Figure 8 (b)). However, if the connection line is considered in the local search process, the simple act of changing the connection point to a new position could achieve a significant improvement in the reduction of the resonant frequency. Figure 8 (c) shows the considerable fall in the resonant frequency from $968.875 \mathrm{MHz}$ to $741.75 \mathrm{MHz}$ when the connection line is moved from segment 21 to segment 55 .

The incorporation of a local search mechanism is a task that requires some time to be studied. The aim is to develop a mechanism that requires as few assessments as possible due to the considerable amount of time required for the NEC software. This is because the local search could multiply the number of solutions generated to be evaluated by the NEC software by a factor determined by how many additional solutions are generated by it.

\section{Conclusions}

This paper described the implementation of an EO algorithm, which was applied to solve a real-world RFID antenna design problem.

The need to extend fitness evaluation to deal with problems that have no available information or data to perform the fitness evaluation for the components of the solution was evident. In response to this requirement, the inseparable fitness evaluation technique was proposed. The implementation of this technique used a pheromone-based mechanism (borrowed from ACO) to assess the contribution of the components of the solution.

A new representation of the solution based on the allocation of segment lines was implemented. With this novel representation, the RFID antenna design was set out as a knapsack problem, which allowed the generation of a new type of antenna design through a modified meander line.

Results show that the EO is an effective and efficient mechanism to find antenna designs with high efficiency for resonant frequencies over $1 \mathrm{GHz}$. The new scheme of the modified meander lines generated by EO has features that the traditional meander line does not, such as an increase in the antenna inductive load. However, it is believed that these same features prevent the new approach from achieving low resonant frequency antenna designs, which are efficiently produced by the ACO algorithms. For this reason, a set of mechanisms, to be implemented as extensions to the EO approach, improving its convergence towards antenna design with low resonant frequencies, were proposed.

Despite the low number of evaluations that could be performed with the EO approach (between 1000 and 3000 evaluations, depending on the size of the instance) compared with the 10000 evaluations performed by previous works, the preliminary results are very promising. A number of potential future works emerge from the proposal. Firstly, a population-based extension of the EO approach is an interesting aspect to be developed, with the aim of increasing the diversity of the solutions. This improvement will be the basis for a parallel implementation of the EO algo- 
rithm, with the objective of reducing the considerable wall-clock time required by the NEC evaluation software. Finally, taking into consideration the good performance achieved by EO to find antenna designs with high efficiencies and by ACO to find antenna designs with low resonant frequencies, a new hybrid heuristic that uses both methods could be implemented. It is believed that with this new hybrid heuristic, enhanced approximated Pareto-front sets could be found.

Acknowledgements The first author is supported by a Bond University Publication Scholarship.

\section{References}

1. Boettcher, S., Percus, A.G.: Extremal optimization: Methods derived from co-evolution. In: Proceedings of the Genetic and Evolutionary Computation Conference, pp. 825-832 (1999)

2. Burke, G., Poggio, A., Logan, J., Rockway, J.: Nec - numerical electromagnetics code for antennas and scattering. In: Antennas and Propagation Society International Symposium, 1979, vol. 17, pp. $147-150$ (1979)

3. Coello Coello, C., Dhaenens, C., Jourdan, L.: Multi-objective combinatorial optimization: Problematic and context. In: Advances in Multi-Objective Nature Inspired Computing, Studies in Computational Intelligence, vol. 272, pp. 1-21. Springer Berlin / Heidelberg (2010)

4. Dorigo, M., Gambardella, L.: Ant colony system: a cooperative learning approach to the traveling salesman problem. IEEE Transactions on Evolutionary Computation 1(1), 53-66 (1997)

5. Finkenzeller, K.: RFID Handbook: Fundamentals and Applications in Contactless Smart Cards, Radio Frequency Identification and Near-Field Communication, Third Edition, john wiley \& sons, inc. edn. John Wiley and Sons (2010)

6. Galehdar, A., Thiel, D., O'Keefe, S., Kingsley, S.: Efficiency variations in electrically small, meander line rfid antennas. In: Antennas and Propagation Society International Symposium, 2007 IEEE, pp. $2273-2276$ (2007)

7. Landt, J.: The history of rfid. Ieee Potentials 24(4), 8-11 (2005)

8. Lewis, A., Randall, M., Galehdar, A., Thiel, D., Weis, G.: Using ant colony optimisation to construct meander-line rfid antennas. In: Biologically-Inspired Optimisation Methods, Studies in Computational Intelligence, vol. 210, pp. 189-217. Springer Berlin / Heidelberg (2009)

9. Lewis, A., Weis, G., Randall, M., Galehdar, A., Thiel, D.: Optimising efficiency and gain of small meander line rid antennas using ant colony system. In: CEC'09: Proceedings of the $11^{\text {th }}$ Congress on Evolutionary Computation, pp. 1486-1492. IEEE Press (2009)

10. Marrocco, G.: Gain-optimized self-resonant meander line antennas for rfid applications. Antennas and Wireless Propagation Letters, IEEE 2(1), 302 -305 (2003)

11. Montgomery, J., Randall, M., Lewis, A.: Differential evolution for rfid antenna design: a comparison with ant colony optimisation. In: In Proceedings of the 13th Annual Genetic and Evolutionary Computation Conference, GECCO 2011, pp. 673-680. ACM (2011)

12. Randall, M., Lewis, A., Galehdar, A., Thiel, D.: Using ant colony optimisation to improve the efficiency of small meander line rfid antennas. In: Third International Conference on e-Science and Grid Computing, e-Science 2007, pp. 345 -351. IEEE Computer Society (2007)

13. Weis, G., Lewis, A., Randall, M., Galehdar, A., Thiel, D.: Local search for ant colony system to improve the efficiency of small meander line rfid antennas. In: Proceedings of the IEEE Congress on Evolutionary Computation, CEC 2008, pp. 1708 -1713. IEEE (2008)

14. Weis, G., Lewis, A., Randall, M., Thiel, D.: Pheromone pre-seeding for the construction of rfid antenna structures using aco. In: Sixth International Conference on e-Science, e-Science 2010, pp. 161-167. IEEE Computer Society, Brisbane, QLD, Australia (2010)

15. Zitzler, E.: Evolutionary Algorithms for Multiobjective Optimization: Methods and Applications. Ph.D. thesis, Swiss Federal Institute of Technology (ETH) (1999) 